\title{
The Royal College diploma program: credential creep or value added?
}

\author{
Amy B. Fraser, MD • Viren N. Naik, MD
}

Received: 26 May 2012/ Accepted: 20 June 2012/Published online: 28 July 2012

(c) Canadian Anesthesiologists' Society 2012

Subspecialty fellowship training in anesthesia undertaken after the completion of residency offers multiple benefits to the profession and to society. Fellowships offer focused teaching in knowledge domains and technical skills that are supplementary and complementary to those taught during residency. In addition, fellows provide academic contributions to their departments, their hospitals, and the profession. Completion of a Canadian fellowship in anesthesia is sometimes awarded with a diploma from a university or hospital, but traditionally this investment in training has not been recognized by an accredited national certifying body. There is an opportunity for that to change.

In 2011, the Royal College of Physicians and Surgeons of Canada (RCPSC) launched its Area of Focused Competence (AFC) diploma certification in subspecialties. This certification program is intended to provide, among other things, recognition to physicians who have special skills that add to, but do not replace, skills utilized during the practice of a primary specialty. According to the RCPSC,

An AFC (diploma) program is a specialized discipline of specialty medicine that addresses a legitimate societal need, but does not meet the Royal College criteria for a specialty, foundation program, or subspecialty. Typically, AFCs (diplomas) represent either a) supplemental competencies that enhance the practice of physicians in an existing discipline, or b) a highly

A. B. Fraser, MD $(\bowtie)$. V. N. Naik, MD

Department of Anesthesiology, The Ottawa Hospital (Civic

Campus), University of Ottawa, 1053 Carling Ave, Suite B302,

Ottawa, ON K1Y 4E9, Canada

e-mail: afraser@toh.on.ca

V. N. Naik, MD

Royal College of Physicians and Surgeons of Canada, Ottawa,

ON, Canada specific and narrow scope of practice that does not meet the criteria of a subspecialty. ${ }^{1}$

This definition applies neatly to many anesthesiologists who have furthered their residency training with a fellowship. Specifically, although they may dedicate a significant amount of their clinical time to subspecialty practice, they are also required to provide generalist anesthesia services. These subspecialty areas are not exclusive to fellowshiptrained anesthesiologists, however, as subspecialty services are often provided by generalist anesthetists.

To date, the RCPCS has established seven AFC diploma programs: Transfusion Medicine, Sports and Exercise Medicine, Adult Echocardiography, Adult Cardiac Electrophysiology, Interventional Cardiology, Cytopathology, and Clinician Educator. There are currently no diploma programs in the field of anesthesiology.

The RCPSC has crafted a robust and generalizable definition of AFCs based on multiple criteria. ${ }^{2}$ According to these criteria, most of the anesthesia fellowship training programs offered at Canadian universities qualify as potential AFC diploma programs. Specific examples include fellowships in cardiovascular, obstetric, pediatric, regional, thoracic, neurosurgical, perioperative medicine, and acute pain anesthesia. The exceptions are chronic pain and critical care medicine, which are considered distinct subspecialties. In essence, AFC diplomas would be applicable to any anesthesia subspecialty for which generalist anesthesiologists have furthered their training academically through education and/or research. They would also be applicable to nonfellowship training or certification that anesthesiologists may undertake, such as certification in transesophageal echocardiography (TEE). Thus, the definition of an AFC is not limited to a traditional one-year fellowship model in terms of its scope and length of training. 
The question we now ask, then, is, "Should Canadian anesthesia programs seek to establish AFC diploma programs in place of the currently offered fellowship programs?" Although a full program evaluation of the AFC diploma would assist in this decision, the data available at this early stage of the AFC initiative are too limited for a comprehensive evaluation.

The aim of this editorial is to provide a discussion using a consumer-oriented evaluation framework, which provides a static (point in time) analysis of process, content, transportability, and effectiveness. ${ }^{3}$ Such an evaluation can illustrate the advantages and disadvantages of AFC diploma programs for anesthesia subspecialties, particularly as compared to the traditional fellowship programs offered by academic centres. We also provide recommendations regarding adoption of AFC diploma programs specific to the specialty.

The basic elements of any successful educational program include clearly stated objectives, sufficient directions, prerequisite knowledge and attitudes, and a match with expertise and existing programs. At present, fellowship programs in anesthesia are designed and managed nearly exclusively by fellowship supervisors within universities and hospitals, with input from their subspecialty colleagues. Currently, there is no centralized body in Canada that oversees academic rigor, the quality of the clinical experience, or program goals and objectives. In the absence of centralized oversight, some fellowships have guided their program curricula to align with subspecialty fellowship requirements in the United States. This is not to say that fellowship programs in Canada are of questionable quality but, rather, that the educational opportunities available in anesthesia fellowships are not generalizable from one program to the next. Although AFC programs in anesthesia do not yet exist, one can imagine a rigorous development process, guided by the RCPSC, that would successfully address all of the necessary educational elements. Once established, an AFC program in anesthesia would provide a standardized "template" for the specific fellowship program to which it applies. This template could then be generalizable to all Canadian anesthesia programs.

In terms of fit with existing programs, clearly there is overlap in the aims and goals of existing fellowship programs and AFC diploma programs - that is, to train competent subspecialist practitioners. However, the existence of AFC programs might also introduce an element of "competition" into fellowship training. A diploma from a well-recognized national college may be attractive to employers and may therefore become attractive to candidates. It could become especially valuable internationally as a portable, recognizable credentialing document. This introduces some uncertainty into the future of subspecialty practice: Will an AFC diploma become required for hiring in Canadian anesthesia departments? What will happen to established subspecialists who do not have AFC certification? What will happen to trainees whose AFC diploma program loses accreditation?

The process by which an AFC diploma is constructed is important. Compared with traditional fellowship programs, AFC diploma programs require participation of the RCPSC for establishment, maintenance, and accreditation. The RCPSC is a well-established educational body with sufficient expertise to provide consultation in this regard. However, significant work will be required from subspecialist anesthesiologists and clinician educators to develop the curriculum for the desired AFC program. Collaboration by multiple centres would be essential to distribute the workload for a project of this scope and to ensure that the curriculum and assessment strategies are generalizable. A coordinated effort that aligns with subspecialty interests and is representative of all of the Fellows of the RCPSC may best be organized through sections of the national specialty society, the Canadian Anesthesiologists' Society.

Any valued credential carries inherent costs for accreditation and certification. There are several areas of expenses, some recurring, when establishing and maintaining an AFC diploma program. ${ }^{4}$ First, the process of applying to establish an AFC diploma program has a onetime cost $(\$ 12,500)$. Second, each institution that elects to offer an AFC diploma program must pay a fixed cost $(\$ 2,000)$ to accredit the program (the schedule of accreditation is not specified). There is also a credentialing fee (\$350) and an assessment fee (\$500) for each candidate. Third, each physician who holds an AFC diploma pays an annual fee (\$425) to continue using the certification professionally. (These costs are those at the time of this writing but are subject to change.) Fourth, there is an indeterminate cost associated with establishing and documenting a curriculum, which requires investing time and expertise. The total cost is not insignificant and may be viewed as a deterrent to establishing AFC diploma programs. These costs would be in addition to the expense of offering a fellowship at an institution. Again, collaboration among centres or through the national specialty society may be a solution to managing these costs, thereby striking an appropriate balance with the benefits of an AFC diploma.

As data on AFC programs become available, it is interesting to observe the organizational, legal, political, and occupational impact of these programs. For example, in the United States, Accreditation Council for Graduate Medical Education (ACGME)-approved fellowship programs exist in parallel with noncertified programs. Programs approved by the ACGME are subject to duty-hour restrictions, practice restrictions, and specific billing regulations, each of 
which can affect the human resources requirements of departments that rely on unrestricted fellow scheduling and the ability of fellows to practice independently. ${ }^{5}$

Another concern is whether an AFC diploma would become an accepted standard for subspecialty practice, thereby influencing strategic staffing and recruitment not only nationally but internationally. Although employer requirements for subspecialty diplomas (whether completed in Canada or internationally) are not yet uniform, one can already observe that "credential creep" is on the rise. This is evidenced in our own specialty, where, for example, some departments require certification in TEE to practice cardiac anesthesiology.

One of the organizational purposes of AFC diploma certification is to prevent the fragmentation of specialties into an increasingly large number of subspecialty "silos," which is of concern to specialists in Canada and internationally. ${ }^{5}$ An important point is the potential stability in quality of care and maintenance of competence that AFC programs offer the global specialty of anesthesia.

It must be noted that this consumer-oriented evaluation is necessarily incomplete. When more data about AFC diploma programs become available, we will be able to compare these programs with existing fellowship programs.

It is possible now, however, to draw some formative conclusions. AFC diploma programs are expensive to initiate and maintain for anesthesia departments, trainees, and diplomates. If it is desirable to establish an AFC diploma program in an anesthesia subspecialty, it may be best done as a collaboration of several academic centres so cost, clinical expertise requirements, and education expertise requirements can be shared.

At the present time, AFC diploma programs have not been widely implemented, with the cost to start these programs likely acting as a deterrent. We recommend that Canadian anesthesia fellowship programs adopt the "educational rigor" of an AFC diploma program until a cost-to-benefit ratio can be examined and the defined costs managed.

\section{Conclusions and recommendations}

We recommend the following specific considerations.

1. Pursuit of a unified, national approach to subspecialty fellowship education, similar to the national curriculum for anesthesia residency training

2. Implementation of a national collaboration within and between subspecialty sections of anesthesia-including the clinical and education sections-to improve and standardize the basic educational elements of our fellowship programs

3. Monitoring the outcomes of AFC diploma programs in comparison to traditional fellowship programs, especially with respect to cost-effectiveness, to determine whether to pursue these programs in the future

Regardless of whether AFC diploma programs become accepted in the field of anesthesia, it is to our advantage as a specialty to examine and implement the principles of the AFC model. It is done with the aim of standardizing the curricula of our fellowship programs using the best educational practices possible.

\section{Le programme de diplômes du Collège royal: inflation des titres de compétence ou valeur ajoutée?}

La formation postdoctorale surspécialisée en anesthésie, entreprise après la fin de la résidence, peut être vue comme offrant de multiples avantages à la profession et à la société. Les formations postdoctorales proposent un enseignement centré sur des domaines de connaissances et des habiletés techniques qui s'ajoute et complète ce qui a été enseigné au cours de la résidence. De plus, ces stagiaires postdoctoraux fournissent souvent des contributions scientifiques à leur département, leur hôpital et, plus généralement, à la profession. Au Canada, l'achèvement d'une formation postdoctorale en anesthésie est parfois reconnu par un diplôme remis par l'université ou l'hôpital; traditionnellement, cet investissement dans la formation n'a pas été reconnu par un organisme national de certification accrédité. Il y a néanmoins une possibilité que cela change.

En 2011, le Collège royal des médecins et chirurgiens du Canada (CRMCC) a lancé son programme d'agrément des diplômes de domaines de compétence ciblée (DCC) pour les surspécialités. Ce programme d'agrément est conçu, notamment, pour reconnaître des habiletés particulières aux médecins qui les possèdent. Ces habiletés s'ajoutent aux compétences utilisées au cours de l'exercice d'une spécialité principale, mais ne les remplacent pas. Selon le CRMCC,

Un programme (diplôme) de DCC est une discipline spécialisée au sein de la spécialité médicale qui répond à un besoin légitime de la société, mais ne correspond pas aux critères du Collège royal pour la définition d'une spécialité, d'un programme de base ou d'une surspécialité. Classiquement, les DCC a) représentent des compétences supplémentaires venant renforcer l'exercice des médecins dans une des disciplines existantes ou $b$ ) couvrent un domaine très précis et étroit de la pratique qui ne répond pas aux critères d'une surspécialité. ${ }^{1}$

La définition ci-dessus s'applique indiscutablement à de nombreux anesthésiologistes qui ont complété la formation 
acquise au cours de leur résidence par une formation postdoctorale. Plus précisément, bien qu'ils puissent consacrer une partie significative de leur activité clinique à la pratique de la surspécialité, il leur est également demandé d'assurer des services d'anesthésie standard. De même, ces domaines de surspécialité ne sont pas exclusivement réservés aux anesthésiologistes ayant suivi une formation postdoctorale, car ces services de surspécialité peuvent souvent être fournis par des collègues généralistes quand cela est nécessaire.

À ce jour, sept programmes de diplôme de DCC ont été mis sur pied: médecine transfusionnelle, médecine du sport et activité physique, échocardiographie de l'adulte, électrophysiologie cardiaque de l'adulte, cardiologie interventionnelle, cytopathologie, et éducateur clinicien. Il n'y a actuellement aucune demande pour des programmes de diplômes dans notre spécialité.

Le CRMCC a brossé une définition solide et généralisable des DCC à l'aide de nombreux critères. $^{2}$ Selon ces critères, la majorité des programmes de formation postdoctorale en anesthésie proposés par les universités canadiennes serait potentiellement admissible au titre de programmes de diplômes de DCC. On pourrait citer, par exemple, les formations postdoctorales en anesthésie cardiovasculaire, obstétricale, pédiatrique, régionale, thoracique, en neuroanesthésie, en médecine périopératoire et en douleur aiguë. Les exceptions incluraient la douleur chronique et la médecine de soins intensifs, qui sont considérés comme des surspécialités particulières. Fondamentalement, les diplômes de DCC peuvent s'appliquer à toute surspécialité d'anesthésie dans laquelle les anesthésiologistes généralistes ont complété leur formation universitaire au travers d'un enseignement et/ou de la recherche. Ils s'appliquent également à des formations autres que postdoctorales ou aux certifications que les anesthésiologistes peuvent entreprendre, tels que l'échocardiographie transœsophagienne (ETO); la définition d'un DCC n'est pas limitée à une formation postdoctorale traditionnelle d'une durée d'un an en termes de portée et de durée de formation.

La question que nous devons maintenant nous poser est la suivante: les programmes canadiens en anesthésie doivent-ils chercher à établir des programmes de diplôme de DCC pour remplacer les programmes de formation postdoctorale actuellement proposés?

Bien qu'une évaluation complète du programme de diplômes de DCC aiderait à prendre une décision, les données dont nous disposons à ce stade précoce de l'initiative des DCC sont encore trop limitées pour permettre une évaluation globale. Cet éditorial proposera donc une discussion en suivant un cadre d'évaluation selon le point de vue du consommateur. Ce cadre a fourni une analyse statique (à un moment donné) du processus, du contenu, de la portabilité et de l'efficacité. ${ }^{3}$ Cette évaluation illustrera les avantages et inconvénients des programmes de diplômes de DCC pour les surspécialités en anesthésie en particulier, comparativement aux programmes de formation postdoctorale traditionnels proposés par les centres universitaires. Nous conclurons en fournissant quelques recommandations concernant l'adoption des programmes de diplômes de DCC propres à la spécialité.

Les éléments fondamentaux de tout programme éducatif réussi incluent des objectifs clairement énoncés, des orientations suffisantes, des connaissances et attitudes préalables et une correspondance avec une expertise et des programmes existants. À l'heure actuelle, les programmes de formation postdoctorale en anesthésie sont conçus et gérés presque exclusivement par des superviseurs de recherche au sein des universités et des hôpitaux, avec un apport de leurs collègues dans leur surspécialité. Il n'existe actuellement au Canada aucun organisme centralisé qui supervise la rigueur scientifique, la qualité de l'expérience clinique, ou les buts et objectifs des programmes. En l'absence de supervision centralisée, certaines formations postdoctorales ont aligné leur contenu sur les exigences de formation en surspécialité existant aux États-Unis. Cela ne veut pas dire que les programmes de formation postdoctorale du Canada sont d'une qualité douteuse, mais plutôt que les possibilités éducatives disponibles en anesthésie au niveau de la recherche postdoctorale ne sont pas généralisables d'un programme à un autre. Même si les programmes de DCC en anesthésie n'existent pas encore, on peut imaginer qu'un processus d'élaboration rigoureux, guidé par le CRMCC, répondrait avec succès à tous les éléments de formation nécessaire. Une fois défini, un programme de DCC fournirait une «trame»standardisée au programme spécifique en formation postdoctorale auquel elle s'applique. Cette trame pourrait ensuite être généralisée à tout programme canadien d'anesthésie.

En matière d'adaptation avec les programmes existants, il existe manifestement un chevauchement des buts et objectifs entre les programmes existants de formation postdoctorale et les programmes de diplôme de DCC destinés à former des praticiens compétents dans une surspécialité. L'existence de programmes de DCC pourrait également introduire un élément de « compétition » dans la formation postdoctorale. Un diplôme remis par un collège reconnu au niveau national peut séduire des employeurs et pourrait donc séduire des candidats. Cela pourrait devenir particulièrement valable sur le plan international en tant que diplômes transférables et identifiables. Cela introduit une certaine incertitude d'un exercice futur de la surspécialité: un diplôme de DCC deviendra-t-il une exigence à l'embauche de professionnels dans les départements d'anesthésie au Canada? 
Qu'adviendra-t-il des praticiens installés exerçant une surspécialité mais ne possédant pas un diplôme de DCC? Et qu'adviendra-t-il des stagiaires dont les programmes de diplômes de DCC perdent leur agrément?

Le processus d'élaboration d'un diplôme de DCC est une affaire sérieuse. Comparativement aux programmes traditionnels de formation postdoctorale, les programmes de diplômes de DCC nécessitent la participation du CRMCC pour leur création, leur maintien et leur agrément. Le CRMCC est un organisme bien implanté dans le monde de l'éducation, ayant une expérience suffisante pour apporter son expertise à ce sujet. Un travail significatif sera toutefois nécessaire de la part des anesthésiologistes pratiquant une surspécialité et de la part des éducateurs cliniciens pour élaborer le contenu des programmes de DCC qui seraient souhaités. La collaboration de nombreux centres serait également essentielle pour répartir la charge de travail pour un projet de cette envergure et s'assurer que le contenu et les stratégies d'évaluation soient généralisables. Un effort coordonné prenant en compte les intérêts de la surspécialité et représentant tous les Associés du CRMCC pourrait être organisé au travers des sections d'une association nationale de spécialistes, la Société canadienne des anesthésiologistes.

Tout titre de valeur entraîne un coût inhérent à l'accréditation et l'agrément. Plusieurs dépenses sont récurrentes dans la mise en place et le maintien d'un programme de diplômes de $\mathrm{DCC}^{4}$ Premièrement, le processus de demande de création d'un programme de diplôme de DCC a au départ un coût de 12500 \$. Deuxièmement, chaque établissement choisissant de proposer un programme de diplômes de DCC doit acquitter un prix fixe de $2000 \$$ pour l'accréditation du programme (le calendrier d'accréditation n'est pas spécifié). Il existe aussi une redevance pour l'obtention du titre (350\$) et pour l'évaluation de chaque candidat (500\$). Troisièmement, chaque médecin détenteur d'un diplôme de DCC devrait payer qu'une cotisation annuelle de 425 \$ pour pouvoir continuer à utiliser son titre sur le plan professionnel. (Ces montants sont exacts au moment de la rédaction de cet éditorial, mais peuvent faire l'objet de modification). Quatrièmement, il existe un coût indéterminé associé à l'élaboration et à la documentation d'un programme, à cause du temps et de l'expertise exigés. Le coût total n'est pas insignifiant et peut paraître dissuasif à la création de programmes de diplômes de DCC. De plus, ces charges s'ajoutent aux dépenses engagées par un établissement pour proposer une formation postdoctorale. Encore une fois, une collaboration entre les centres ou par le biais des associations nationales de spécialistes peut-être une façon de gérer ces frais afin de parvenir à un équilibre satisfaisant avec les avantages procurés par un diplôme de DCC.
Au fur et à mesure que des données sur les programmes de DCC deviendront disponibles, il sera intéressant d'observer l'impact organisationnel, juridique, politique et professionnel de ces programmes. Aux États-Unis, par exemple, des programmes de formation postdoctorale approuvés par l'ACGME (Accreditation Council for Graduate Medical Education) existent parallèlement à des programmes non agréés. Les programmes approuvés par l'ACGME sont soumis à des restrictions sur les heures de garde, des restrictions touchant l'exercice professionnel et une réglementation spécifique sur la facturation qui peuvent, toutes, affecter les besoins en ressources humaines de départements qui reposent sur une disponibilité sans restriction des stagiaires postdoctoraux et la possibilité pour ces derniers d'exercer de façon indépendante. ${ }^{5}$

Une autre préoccupation sera de savoir si un diplôme de DCC deviendra une norme acceptée pour l'exercice de la surspécialité et influencera les stratégies de recrutement et d'affectation du personnel, non seulement du point de vue national, mais aussi international. Tandis que les exigences des employeurs concernant les diplômes de surspécialité (que ce soit au Canada ou ailleurs dans le monde) ne se sont pas encore uniformisées, il est déjà possible d'observer un accroissement de «l'inflation des titres de compétences ». Cela est visible dans notre propre spécialité, où une certification en ETO est requise par certains départements pour pouvoir, par exemple, exercer en anesthésiologie cardiaque.

L'un des objectifs organisationnels de l'agrément des diplômes de DCC est de prévenir la fragmentation des spécialités en un nombre toujours croissant de « silos » de surspécialités, ${ }^{1}$ ce qui constitue une préoccupation au Canada et ailleurs dans le monde. ${ }^{5}$ La stabilité potentielle de la qualité des soins et le maintien des compétences que les programmes de DCC pourraient offrir à l'ensemble de la spécialité de l'anesthésie seront importants à suivre.

Il est important de noter que cette évaluation du point de vue du consommateur est nécessairement incomplète. Une comparaison entre ces programmes et les programmes de formation postdoctorale existants sera nécessaire quand nous disposerons de davantage de données sur les programmes de diplômes de DCC.

Il est toutefois possible de dessiner quelques grandes lignes en guise de conclusion. Les programmes de diplômes de DCC coûteront cher à mettre en place et à maintenir, à la fois pour les départements d'anesthésie et pour les stagiaires ou diplômés. S'il est souhaitable d'établir un programme de diplôme de DCC pour une surspécialité d'anesthésie, cela pourra être fait au mieux grâce à une collaboration entre plusieurs centres universitaires de façon à ce que son coût, les besoins d'expertise clinique et d'expertise éducative puissent être répartis. 
Les programmes de diplômes de DCC n'ont pas encore été largement mis en œuvre à ce jour. Les obstacles et coûts de création de ces programmes seront probablement dissuasifs pour des disciplines de surspécialité. Nous recommandons que les programmes canadiens de formation postdoctorale en anesthésie adoptent au moins la rigueur universitaire d'un programme de diplôme de DCC jusqu'à ce qu'un rapport coût-bénéfice puisse être examiné et les coûts définis puissent être gérés.

\section{Conclusions et recommandations}

Nous préconisons les recommandations spécifiques suivantes:

4. Poursuite d'une approche nationale unifiée de la formation postdoctorale de la surspécialité, semblable au programme national pour la formation des résidents en anesthésie.

5. Mise en œuvre d'une collaboration nationale au sein des sections des surspécialités d'anesthésie et entre celles-ci, y compris les sections cliniques et universitaires, pour améliorer et normaliser les éléments éducatifs de base de nos programmes de formation.

6. Surveillance des résultats des programmes de diplômes de DCC par rapport aux programmes traditionnels de formation postdoctorale, tout particulièrement quant à leurs rapports efficacité-coût pour déterminer s'il y a lieu de poursuivre ces programmes à l'avenir.
Que les programmes de diplômes de DCC finissent ou non par être acceptés en anesthésie, nous avons intérêt - en tant que spécialité - à étudier de près et à mettre en œuvre les principes du modèle de DCC afin de normaliser les contenus en se basant sur les meilleures pratiques éducatives pour nos programmes de formation postdoctorale.

Financial support None.

Conflict of interest None declared.

\section{References}

1. The Royal College of Physicians and Surgeons of Canada. Area of Focused Competence AFC (Diploma) Program. Available from URL: http://www.royalcollege.ca/shared/documents/specialty_recognition/ application_form_diplomas_e.docx (accessed May 2012).

2. The Royal College of Physicians and Surgeons of Canada. Criteria for Areas of Focused Competence (Diplomas), Fundamentals \& Special Interest Groups of Medical Activity (SIGMAs) - May 2010. Available from URL: http://www.royalcollege.ca/shared/documents/ specialty_recognition/diploma_criteria.pdf (accessed May 2012).

3. Fitzpatrick JL, Sanders JR, Worthen BR. Program Evaluation: Alternative Approaches and Practical Guidelines. 3rd ed. Boston: Allyn \& Bacon; 2003.

4. The Royal College of Physicians and Surgeons of Canada. How to Apply for Recognition of an Area of Focused Competence (AFC) Diploma Completing Your Application. Available from URL: http://www.royalcollege.ca/public/credentials/afc_program/afc_ application (accessed May 2012).

5. Desjardins $G$, Calahan $M K$. Subspecialty accreditation: is being special good? Curr Opin Anaesthesiol 2007; 20: 572-5. 